\title{
Design and Verification of Aeroengine Rotor Speed Controller Based on U-LADRC
}

\author{
Jiajie Chen, Jiqiang Wang $\mathbb{D}^{D}$, Yunxiao Liu, and Zhongzhi Hu \\ Jiangsu Province Key Laboratory of Aerospace Power Systems, Nanjing University of Aeronautics and Astronautics, Nanjing, \\ Jiangsu 210016, China \\ Correspondence should be addressed to Jiqiang Wang; jiqiang_wang@hotmail.com
}

Received 17 December 2019; Revised 9 March 2020; Accepted 20 April 2020; Published 22 May 2020

Guest Editor: Jian Huang

Copyright (c) 2020 Jiajie Chen et al. This is an open access article distributed under the Creative Commons Attribution License, which permits unrestricted use, distribution, and reproduction in any medium, provided the original work is properly cited.

Due to the harsh working environment, engine electronic controller (EEC) has limited computing power. Many advanced control algorithms are difficult to be applied in practice because of complexity of calculation. In this paper, a novel aeroengine transient-speed controller with low algorithm complexity is designed by combining linear parameter varying (LPV) model with U-control theory. Aiming at restraining bad performance influence caused by possible disturbance in cruise, linear active disturbance rejection control (LADRC) compensation is integrated as the U-LADRC controller. This new controller is verified in both the digital simulation platform and hardware-in-the-loop (HIL) platform. The experimental results of the HIL platform show that the U-LADRC control algorithm meets the real-time performance of the EEC in the actual aeroengine. It has good transition state control performance and good steady-state antidisturbance ability, which ensures the smooth operation of the engine in the steady state and has a good practical application prospect.

\section{Introduction}

Aeroengine is a complex time-varying nonlinear thermodynamic system, and its dynamic performance varies with the change of engine thrust and flight conditions. Because of the harsh environment and many kinds of disturbances in real working state, the engine electronic controller (EEC) has finite computational ability with around $20 \mathrm{~ms}$ calculation period (control algorithms cost $\leq 5 \mathrm{~ms}$ ) [1]. Therefore, the characteristics of aeroengine speed controller with practical application potential should at least include the following:

(1) Good transient and steady-state control performance

(2) Simple structure

(3) Low algorithm complexity

(4) Wide range of flight envelope application

(5) Good antidisturbance ability

Up to now, only PID and LQG/LTR control method can be applied to the real EEC in aeroengine maturely [2]. But these methods still have deficiencies such as antidisturbance ability of PID is limited and weight matrix of LQG/LTR is difficult to choose. Due to the fact that advanced control algorithms such as intelligent control algorithms usually have high algorithm complexity, few of them can have practical application potential. Therefore, it is very important to create a novel control method which can meet the previous requirements $1-5$.

These years, a variable scheduling controller design method based on the linear parameter varying model (LPV) has received more and more attention. In this control method, a LPV model capable of characterizing the dynamic performance of an aeroengine is first established; then the corresponding linear controller is designed directly according to the scheduling parameters in the LPV model, without interpolation and switching between the controllers. The widely used controller design method based on LPV model relies on Lyapunov function to solve linear matrix inequality (LMI) on the whole control envelope parameter trajectory, which has high complexity and difficulty in solving. It is hard for realistic application.

Aiming at this problem, this paper focuses on the simplification of the algorithm complexity and the improvement of the antidisturbance performance. The LPV 
model is combined with the U-control theory and the active disturbance rejection control (ADRC) theory to design a steady and transient speed controller with good real-time performance. U-control theory was originally developed by Zhu and Guo [3] in 2002. The core idea of the theory is to transform the nonlinear controlled object into a U-model structure oriented to control and then realize the online solution of the dynamic inverse of the controlled object. $\mathrm{U}$-model structure is a polynomial structure that comprises time-varying system parameters. It establishes a simple general mapping which can transform almost all smooth nonlinear input-output dynamic object models into designable structures of linear control methods, simplifying the design of the controller. After more than ten years of development [4], the nonlinear control method based on U-model has been applied to predictive control [5], internal model control [6], adaptive control [7, 8], and so on. Active disturbance rejection control (ADRC) is a control method proposed by Han in the 1990s that can solve uncertain systems with a large range of complex structures [9]. The main idea is to eliminate the amplifying effect of differentiation on high-frequency disturbance by using a differential tracker, rearrange the transition process, and make full use of the role of differential feedforward. Without relying on the model, the disturbances and system uncertainties can be estimated online by extended state observer (ESO) and the corresponding compensation of control parameters can be performed in real time, which eliminates the influence of disturbances and system uncertainties on instruction tracking to the greatest extent. But the ADRC method needs to use complex algorithms and adjust too many parameters. In order to solve this problem, Gao proposes the linear active disturbance rejection control (LADRC) method [10]; it linearizes part of the control structure and reduce the number of adjusting parameters and algorithms greatly. Compared with the ADRC method, LADRC can also achieve good control results and save unnecessary engineering calculation time, which is more conducive to the realistic application. To deal with the system uncertainties, many control methods are combined with disturbance observer (DO) $[11,12]$ or extended state observer (ESO) [13] to improve their antidisturbance performance. Therefore, based on the principle of its linear ESO, LADRC can be used as a disturbance compensator for mismatched uncertainties aeroengines may meet during the flight mission.

In this paper, a gear turbofan (GTF) engine componentlevel nonlinear model is taken as the research object. First, the LPV model is established based on the nonlinear engine model. Then, the LPV model is converted into a U-model structure, and a dynamic inverse solver module is designed. A linear controller with a fixed structure was designed in series and connected to the closed-loop speed control loop to form a transient speed controller (U-controller). In order to solve the problem of poor antidisturbance ability of the controller, LADRC disturbance compensation module is introduced to compensate the effect of the aeroengine caused by disturbance in advance in steady state. The digital simulation results show that the U-LADRC rotor speed controller is successfully applied to a wide range of dynamic speed control near the cruise steady state. At the same time, it has a good disturbance suppression effect against the atmospheric disturbance and power extraction disturbance that may exist during the cruise steady state. Finally, the hardware-in-the-loop (HIL) simulation platform was used to verify the real-time performance of the control algorithm in a real EEC. The results show that the control effect is basically the same as the digital simulation result and meets the real-time requirements of the real EEC. Besides, the U-LADRC controller significantly improves the antidisturbance performance under steady-state conditions after the introduction of the LADRC compensator.

This paper is organized as follows: U-control concept, principle of LADRC, and aeroengine LPV model are introduced in Section 2. The design method of aeroengine U-LADRC rotor speed controller is introduced in Section 3, which is the core part in this paper. Digital simulation test is carried out to verify the transient-state control performance and steady-state antidisturbance ability preliminarily in Section 4. Section 5 shows the verification results of U-LADRC controller in the HIL platform.

\section{Preliminaries}

2.1. U-Control Concept. U-control method provides a kind of linear controller design method for nonlinear objects based on U-model structure. U-model uses time-varying parameter polynomial to represent a large class of smooth nonlinear systems; it consists of the power series of the current input. Consider a single-input and single-output (SISO) polynomial $\mathrm{U}$-model with a triplet of $(y(t), \lambda(t), u(t))$, the system can be expressed as follows [14]:

$$
\stackrel{(M)}{y}(t)=\sum_{j=0}^{J} \lambda_{j}\left(Y_{M-1}, U_{N-1}, \Theta\right)(\stackrel{(N)}{u}(t))^{j}, \quad M>N,
$$

where

$$
\begin{aligned}
& Y_{M-1}=[\stackrel{(M-1)}{y}(t), \ldots, y(t)] \in R^{M}, \\
& U_{N-1}=\left[\begin{array}{c}
(N-1) \\
u
\end{array}(t), \ldots, u(t)\right] \in R^{N},
\end{aligned}
$$

$\stackrel{(M)}{y}(t) \in R$ and $\stackrel{(N)}{u}(t) \in R$ are the $M$ th and $N$ th orders of derivatives of output $y(t)$ and input $u(t)$, respectively. $\lambda_{j}(t) \in R$ is the time-varying parameter absorbing all the other inputs $Y_{M-1}$, outputs $U_{N-1}$, and the coefficients $\Theta$ associated with the input $(\stackrel{(N)}{u}(t))^{j}$.

The dynamic inversion is to obtain the input $u(t)$ from $\stackrel{(M)}{y}(t)$. For a given output $\stackrel{(M)}{y}(t), u(t)$ is solved by

$$
\stackrel{(N)}{u}(t) \in \stackrel{(M)}{y}(t)-\sum_{j=0}^{J} \lambda_{j}\left(Y_{M-1}, U_{N-1}, \Theta\right)(\stackrel{(N)}{u}(t))^{j}=0 .
$$

Simple example: consider a linear state-space model,

$$
\begin{aligned}
{\left[\begin{array}{l}
\dot{x}_{1} \\
\dot{x}_{2}
\end{array}\right] } & =\left[\begin{array}{cc}
0 & 1 \\
-1 & -1
\end{array}\right]\left[\begin{array}{l}
x_{1} \\
x_{2}
\end{array}\right]+\left[\begin{array}{l}
2 \\
1
\end{array}\right] u, \\
y & =\left[\begin{array}{ll}
1 & 0
\end{array}\right]\left[\begin{array}{l}
x_{1} \\
x_{2}
\end{array}\right] .
\end{aligned}
$$


Equation (4) can be expressed as the U-model structure:

$$
\dot{y}(t)=\lambda_{0}(t)+\lambda_{1}(t) u(t)
$$

where $\lambda_{0}(t)=3 x_{1}(t)+x_{2}(t)$ and $\lambda_{1}(t)=2$.

The dynamic inversion can be calculated as

$$
u(t)=\frac{\dot{y}(t)-\lambda_{0}(t)}{\lambda_{1}(t)} .
$$

The main idea of $\mathrm{U}$-control concept is that the dynamic inversion of U-model can be served as the inverse model of the controlled nonlinear system. It is connected in series in the control system loop as the part of the controller. In this way, the remaining controller design problem for a nonlinear system can be transformed into a very simple problem. Only a linear controller for the controlled object "1" is needed to design. Figure 1 shows the closed-loop structure of U-control.

In Figure $1, G_{c 1}$ is a linear invariant controller, $G_{P}^{-1}$ is served as the dynamic inverse of the plant $G_{p}$, and $G_{c 1}$ and $G_{p}^{-1}$ are designed separately to form the resultant controller $G_{c}=G_{c 1} G_{p}^{-1}$.

\subsection{Linear Active Disturbance Rejection Control (LADRC).} The main feature of ADRC is to estimate the unknown disturbance and the dynamic uncertainty of the system online by designing the extended state observer (ESO), so that the disturbance and unknown dynamic compensation can be performed in real time, minimizing the bad effects of disturbances and unknown dynamics on instruction tracking.

Traditional ADRC controller has many parameters which are difficult to adjust, and too many nonlinear terms are adverse to engineering application. Therefore, this paper adopts a LADRC compensator for disturbance compensation. The LADRC controller is simplified from the ADRC controller. Tracking differentiation (TD) is omitted. The nonlinear function part of the extended state observer (ESO) is changed to linear ESO, and the nonlinear PD control is changed to linear PD control. The structure of LADRC is shown in Figure 2.

The second-order controlled object is taken as an example:

$$
\left\{\begin{array}{l}
x^{\prime \prime}=f\left(x, x^{\prime}, d, t\right)+\mathrm{Bu}, \\
y=x
\end{array}\right.
$$

where $x, x^{\prime}, x^{\prime \prime}$, and $y$ are, respectively, the system states and their first and second derivatives. The principle of LESO is

$$
\left\{\begin{array}{l}
\dot{z}_{1}=z_{2}-\beta_{1}\left(z_{1}-y\right), \\
\dot{z}_{2}=z_{3}-\beta_{2}\left(z_{1}-y\right)+\mathrm{Bu}, \\
\dot{z}_{3}=-\beta_{3}\left(z_{1}-y\right),
\end{array}\right.
$$

where $\beta_{1}, \beta_{2}$, and $\beta_{3}$ are adjustable parameters in LESO, $z_{1}, z_{2}$, and $z_{3}$ represent the estimations of the output $y$, the differential of $y$, and the unknown disturbance of system, respectively. By compensating control parameters, the system can be linearized into an integrator series structure.

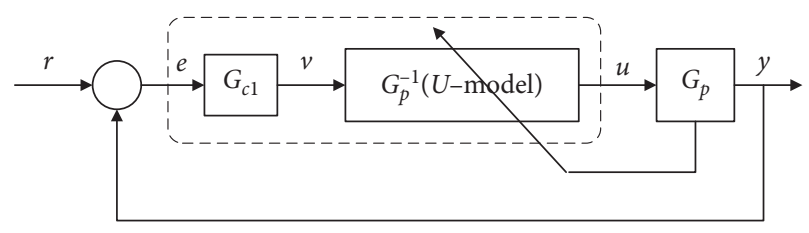

Figure 1: Closed-loop structure of U-control.

LESO can accurately observe various internal and external disturbances and introduce them into the control loop for compensation, so as to achieve the purpose of antidisturbance. The reliability of LESO can be proved by frequency-domain analysis method [15] and matrix norm theory [16].

The control law in LADRC is

$$
\left\{\begin{array}{l}
u_{0}=k_{p}\left(y_{k p}-z_{1}\right)-k_{d} z_{2}, \\
u=\frac{\left(u_{0}-z_{3}\right)}{B},
\end{array}\right.
$$

where $k_{p}, k_{d}$, and $B$ are the control parameters of PD link. Adjustable parameters determine the quality of the observer and also greatly affect the antidisturbance ability of the control system. To maintain the stability of LADRC system, $\beta_{1}, \beta_{2}, \beta_{3}, k_{p}$, and $k_{d}$ can be selected by the rule [17] as follows:

$$
\left\{\begin{array}{l}
\beta_{1}=3 \omega_{o}, \\
\beta_{2}=3 \omega_{o}^{2}, \\
\beta_{3}=\omega_{o}^{3}, \\
k_{p}=\omega_{c}^{2}, \\
k_{d}=2 \omega,
\end{array}\right.
$$

where $\omega_{o}$ and $\omega_{c}$ are observer bandwidth and controller bandwidth. By selecting appropriate bandwidths, the LADRC system can keep a good stability [15].

2.3. Aeroengine LPV Model. This paper researches on a Geared Turbofan (GTF) engine, as shown in Figure 3. GTF engine represents the next generation of high-efficiency engines. In the traditional turbofan engine, the fan is directly driven by the low-pressure shaft, so the fan, LPC, and LPT cannot work in their own best rotational speeds at the same time. By using a gear box, the GTF engine solves this problem of speed contradiction. The fan can work in ideal low speed and the LPT can keep high-speed rotation, which reduces the engine noise and fuel consumption. Therefore, carrying out researches on designing the control system for this advanced engine is very necessary.

The engine model used in this research is an advanced GTF engine nonlinear component-level model provided by Toolbox for the Modeling and Analysis of Thermodynamic Systems (T-MATS) [18].

The GTF model has 3 main control parameters: fuel flow $W_{f}$, variable bleed valve (VBV), and variable-area fan nozzle (VAFN). VBV can transfer the air flow from the LPC outlet to the bypass to prevent the LPC from stalling. 


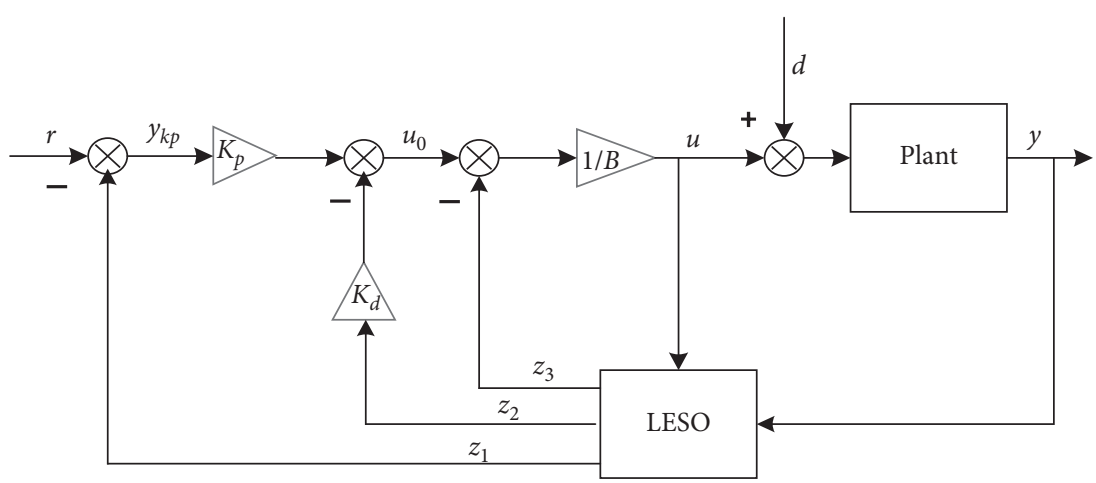

FIgURE 2: The structure of LADRC.

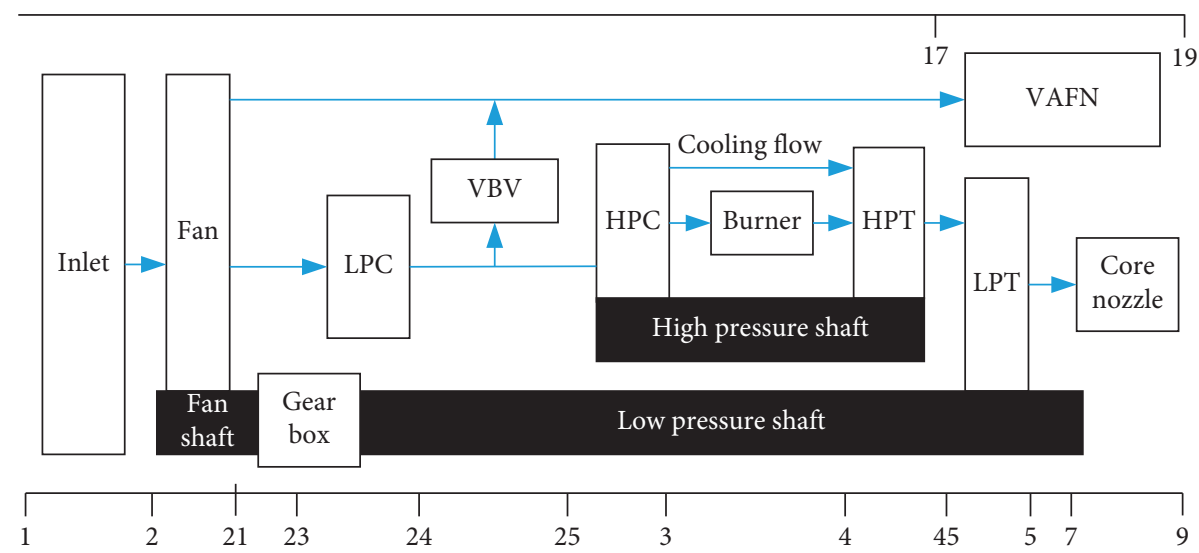

FIgURE 3: The structure of GTF engine.

VAFN maintains a specific pressure ratio at a given corrected flow rate and rotor speed to make the fan keep the optimal performance at the operating point. These two control variables are calculated by a schedule related to the flying Mach number Ma and the fan speed $N_{f c}$; they are not in the closed-loop control. The fuel flow $W_{f}$ is the main control amount obtained through the closed-loop control of the control system. In the SISO model studied in this paper, the input is the fuel flow rate $W_{f}$ and the output is the LP rotor speed $N_{L}$. Based on the component-level nonlinear model, the LPV model is established off-line in classical Jacobian linearization. Mathematical expression of the engine nonlinear model is shown below:

$$
\begin{aligned}
& \dot{x}=f(x, u), \\
& y=g(x, u),
\end{aligned}
$$

where $f(\cdot)$ and $g(\cdot)$ are continuously differentiable. A steady-state point $\left(x_{0}, u_{0}\right) \in\{(x, u) \mid f(x, u)=0\}$ is selected, and Taylor expansion is carried out ignoring the minor terms of the second order and above; we can obtain

$$
\begin{aligned}
& f(x, u) \approx f\left(x_{0}, u_{0}\right)+\left.\frac{\partial f}{\partial x}\right|_{\left(x_{0}, u_{0}\right)} \Delta x+\left.\frac{\partial f}{\partial u}\right|_{\left(x_{0}, u_{0}\right)} \Delta u, \\
& g(x, u) \approx g\left(x_{0}, u_{0}\right)+\left.\frac{\partial g}{\partial x}\right|_{\left(x_{0}, u_{0}\right)} \Delta x+\left.\frac{\partial g}{\partial u}\right|_{\left(x_{0}, u_{0}\right)} \Delta u,
\end{aligned}
$$

namely, the linearized model which can describe the dynamic process at neighbourhood around a steady-state point is

$$
\begin{aligned}
& \Delta \dot{x}=A \Delta x+B \Delta u, \\
& \Delta y=C \Delta x+D \Delta u
\end{aligned}
$$

To study the rotor speed dynamic performance around the cruise condition, the input $u$ is the fuel flow, and the output $y$ represents LP/HP shaft rotational speeds. In order to avoid the ill-condition of the system matrix, the input and output parameters are normalized. The normalized fuel flow $\mathrm{PW}_{f}$ is taken as an example:

$$
\mathrm{PW}_{f}=\frac{W_{f}}{W_{f d}},
$$

where $W_{f d}$ is the fuel flow in steady-state design point in cruise.

A series of normalized state-space models of steady-state points around the cruise are taken to establish the LPV model:

$$
\begin{aligned}
& \dot{x}=A(\theta) x+B(\theta) u, \\
& y=x,
\end{aligned}
$$

where $x \in R^{n}$ is the state vector $\left[\begin{array}{lll}\Delta \mathrm{PN}_{L} & \Delta \mathrm{PN}_{H}\end{array}\right]^{T} ; y \in R^{n}$ is the output vector $\left[\begin{array}{ll}\Delta \mathrm{PN}_{L} & \Delta \mathrm{PN}_{H}\end{array}\right]^{T} ; u \in R$ is the control parameter fuel flow variation $\Delta \mathrm{PW}_{f}$; and $\theta \in R$ is the 
scheduling parameter normalized high-pressure rotational speed $\mathrm{PN}_{H}$. Linear interpolation scheduling method is adopted.

In this study, for the SISO system from fuel flow to LP rotor speed, the LPV relationship between the variation of them is

$$
\Delta \mathrm{PN}_{L}=a_{11}(\theta) \Delta \mathrm{PN}_{L}+a_{12}(\theta) \Delta \mathrm{PN}_{H}+b_{1}(\theta) \Delta \mathrm{PW}_{f} .
$$

\section{Design of Aeroengine U-LADRC Controller}

Affected by the harsh environment and changes in its own operating mode, aeroengines often encounter various disturbances (e.g., atmospheric disturbance, power extraction disturbance) during steady-state operation in cruise. At this time, problems such as mismatch of the LPV model may be caused, resulting in large fluctuation in the rotor speed and poor antidisturbance performance. Aiming at this problem, a LADRC fuel flow compensation module is added to improve the antidisturbance performance of the U-control algorithm. The design process of aeroengine U-LADRC rotor speed controller can be divided into two parts: design of the U-controller and design of the LADRC fuel flow compensation.

3.1. Design of the U-Controller. The U-controller is the core part in U-LADRC rotor speed controller which guarantees the based transient and steady-state control performance in cruise. Based on Figure 1, the design process is introduced in the following:

3.1.1. Convert LPV Model into U-Model Structure. Converting LPV model into first-order U-model structure,

$$
\Delta \dot{\mathrm{N}}_{L}=\lambda_{1} \Delta \mathrm{PW}_{f}+\lambda_{0},
$$

where $\lambda_{1}=b_{1}(\theta), \lambda_{0}=a_{11}(\theta) \Delta \mathrm{PN}_{L}+a_{12}(\theta) \Delta \mathrm{PN}_{H}$, and $\lambda_{1}$ and $\lambda_{2}$ are time-varying coefficients.

3.1.2. Calculate Dynamic Inversion $G_{p}^{-1}$ Based on U-Model. The inversion of $\mathrm{U}$-model can be expressed as

$$
\Delta \mathrm{PW}_{f}=\frac{\left(\Delta \dot{\mathrm{PN}}_{L}-\lambda_{0}\right)}{\lambda_{1}} .
$$

Therefore, the real control parameter is

$$
\begin{aligned}
W_{f_{-} U} & =W_{f_{s t}}(\theta)+\Delta W_{f} \\
& =W_{f_{s t}}(\theta)+\Delta P W_{f} \cdot W_{f d},
\end{aligned}
$$

where $W_{f s t}(\theta)$ is the fuel flow baseline corresponding to scheduling parameter $\theta . W_{f d}$ is the fuel flow design value which is used for normalizing fuel flow values.

3.1.3. Design of the Linear Invariant Controller $G_{c 1}$. Based on U-control concept, in perfect match the $G_{p}^{-1} G_{p}=1$, the design problem can be simplified as designing a controller for a controlled object "1." There are many methods to design linear controller. In U-control condition, the easiest linear controller can be a simple gain module, or a first-order damp element. For application of engineering, here a first-order damp element is chosen. Because the LPV model is normalized, the linear controller is designed as follows:

$$
G_{c 1}=\frac{K}{T s+1},
$$

where $K=\left(1 / N_{L_{-} s}\right), N_{L_{-} s}$ is the LP rotor speed steady-state design point in cruise. It is easy to prove that $G_{c l}$ and the closed-loop transfer function $G=\left(G_{c l} / 1+G_{c l}\right)$ are stable.

Considering the model error "mismatch," the real plant can be described as

$$
G_{p}^{\prime}=G_{p}+\Delta,
$$

where $G_{p}^{-1} G_{p}^{\prime} \neq 1$. Defining the uncertainty of the model,

$$
E=\frac{\Delta}{G_{p}} \text {. }
$$

Whether it is atmospherics disturbance or power extraction disturbance discussed in the research, $|E|$ is impossible to be too large. At least, $|E|<1$ is undisputed. Therefore, the following conditions are satisfied:

(a) $G=\left(G_{c l} /\left(1+G_{c l}\right)\right)$ is stable

(b) $|G(j w) \cdot E(j w)|<1$

Based on the small gain theorem [19], the U-controller closed-loop system can keep stable.

3.2. Design of the LADRC Fuel Flow Compensation. LADRC fuel flow compensation takes the difference between the actual speed and the current ideal steady-state speed as the input and uses the calculated fuel correction value as the output. Take LADRC compensation control target $r$ equal to 0 to reduce the fluctuation of LP rotor speed when the disturbance exists. Actual fuel flow at this time can be calculated as

$$
W_{f}=W_{f_{-} U}+W_{f_{-} \mathrm{LADRC}}
$$

where $W_{f_{-} \text {LADRC }}$ is calculated based on $(8) \sim(10)$. In summary, the U-LADRC rotor speed control structure is shown in Figure 4. The U-LADRC rotor speed controller consists of a U-controller and a LADRC disturbance compensator. When the aeroengine is in the transient-state process, the LADRC disturbance compensator does not work and the $\mathrm{U}$-controller guarantees good performance of the transientstate response. When the aeroengine is in the steady-state operating condition, the LADRC disturbance compensator is enabled to restrain the bad effects caused by various possible disturbances.

\section{Simulation in MATLAB/Simulink}

In order to verify the control and antidisturbance performance of the U-LADRC algorithm, the verification 


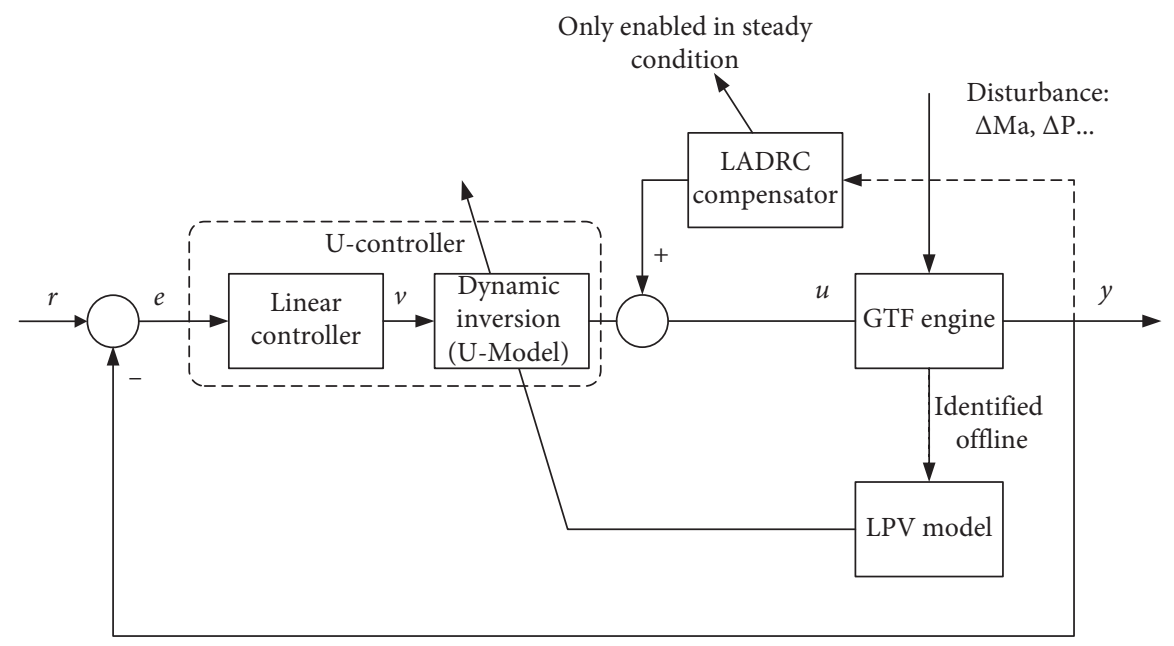

FIgURE 4: The structure of U-LADRC controller.

processes in two cases are designed on the MATLAB/ Simulink digital simulation platform.

4.1. Transient-State Process. In the cruise condition $(H=35000 \mathrm{ft}, \mathrm{Ma}=0.8)$, the aeroengine is simulated from normal cruise point $\left(N_{L}=6777 \mathrm{rpm}\right) \longrightarrow$ lower cruise point $\left(N_{L}=6551 \mathrm{rpm}\right) \longrightarrow$ normal cruise point $\longrightarrow$ upper cruise point $\left(N_{L}=6915 \mathrm{rpm}\right) \longrightarrow$ normal cruise point transient state process, command speed changing curve and U-controller transient state control effect is shown in Figure 5. The fuel flow changing curve is shown in Figure 6. Simulation results show that the steady-state error is 0 . The algorithm of $\mathrm{U}$-controller can be preliminarily verified effectively.

4.2. Steady-State with Disturbance. In the normal cruise condition $\left(H=35000 \mathrm{ft}, \mathrm{Ma}=0.8\right.$, and $\left.N_{L}=6777 \mathrm{rpm}\right)$, atmospheric disturbance and power extraction disturbance are used for simulating the disturbance suppression effect of the U-LADRC controller. Besides, the U-controller without the LADRC disturbance compensator is used as a comparison.

4.2.1. Atmospherics Disturbance. In this paper, Kopasakis's atmospheric turbulence model is used [20]. The combination of sine curves of unit amplitude is used to obtain the atmospheric disturbance model, which acts on the inlet of the GTF engine model. Atmospheric disturbance can cause changes in Mach number, temperature, and pressure; then it may lead to aeroengine performance becoming worse. The Kopasakis atmospheric disturbance model is shown in Figure 7.

To study the antidisturbance effect of U-LADRC controller on the LP rotor speed under the atmospheric disturbance, disturbance effects of static temperature, static pressure, and Mach number are added to the inlet of the GTF engine model at the same time from 20 to $30 \mathrm{~s}$. These disturbances are shown in Figure 8.

The antidisturbance effect of U-LADRC controller and U-controller without LADRC disturbance

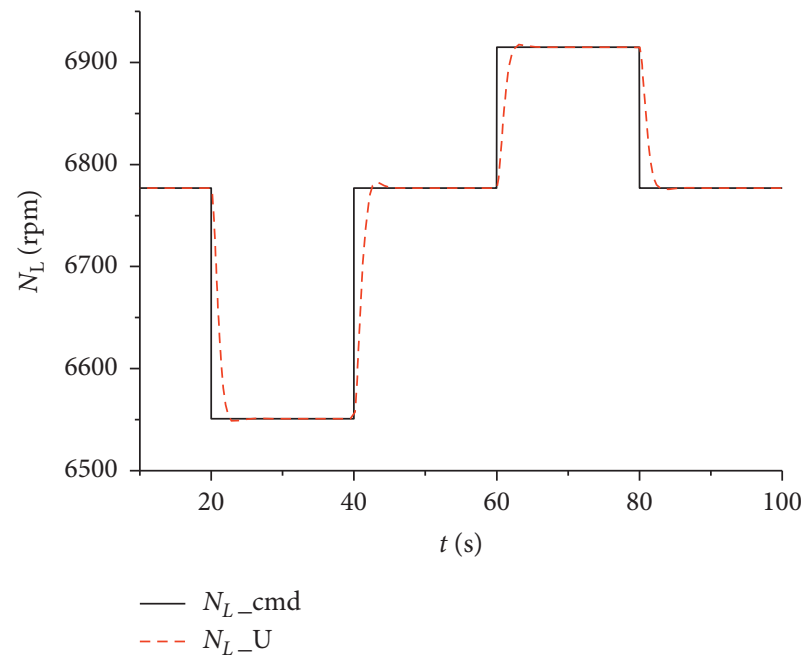

FIGURE 5: U-controller transient-state control effect.

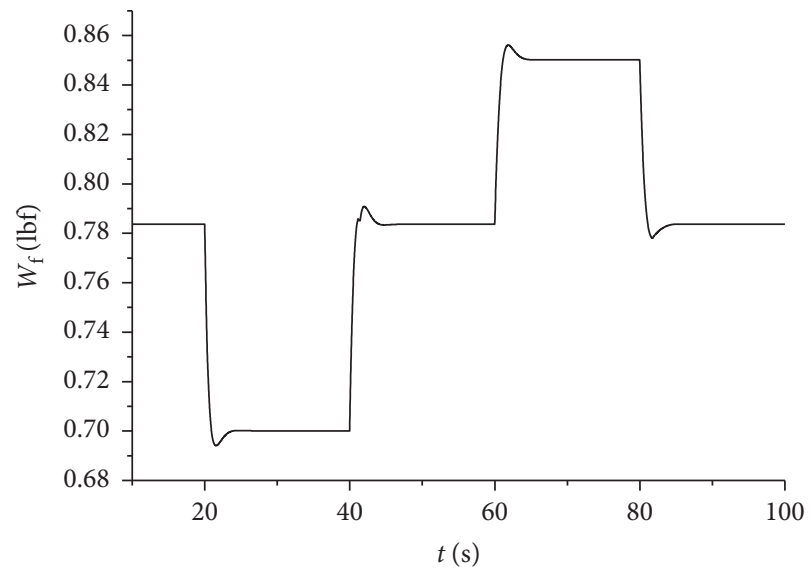

Figure 6: Fuel flow changing curve.

compensator is compared in Figures 9 and 10. We can see that U-LADRC controller leads to a better antidisturbance effect with the help of advanced fuel flow 


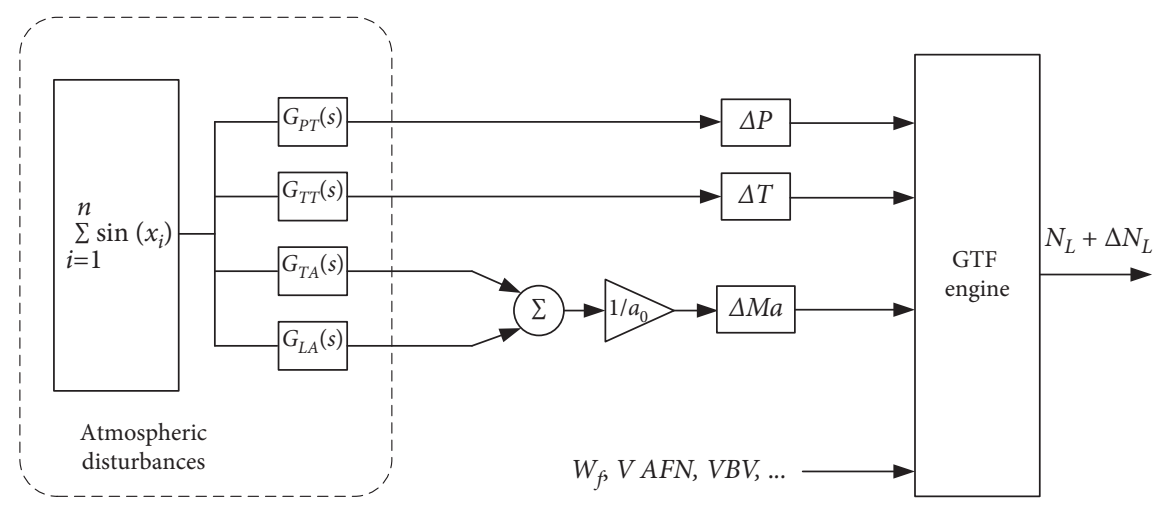

FIgURE 7: Kopasakis's atmospheric disturbance model.
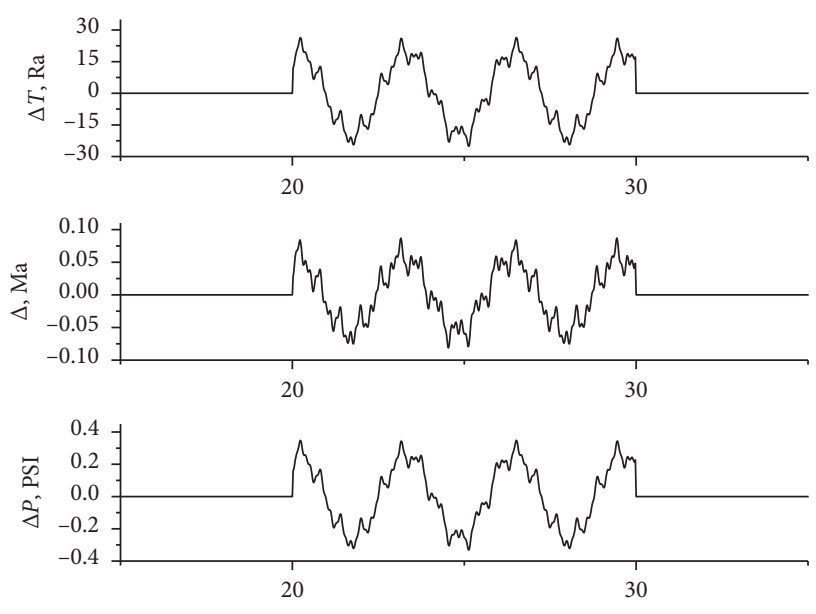

$t(\mathrm{~s})$

Figure 8: Atmospheric disturbances added in the inlet.

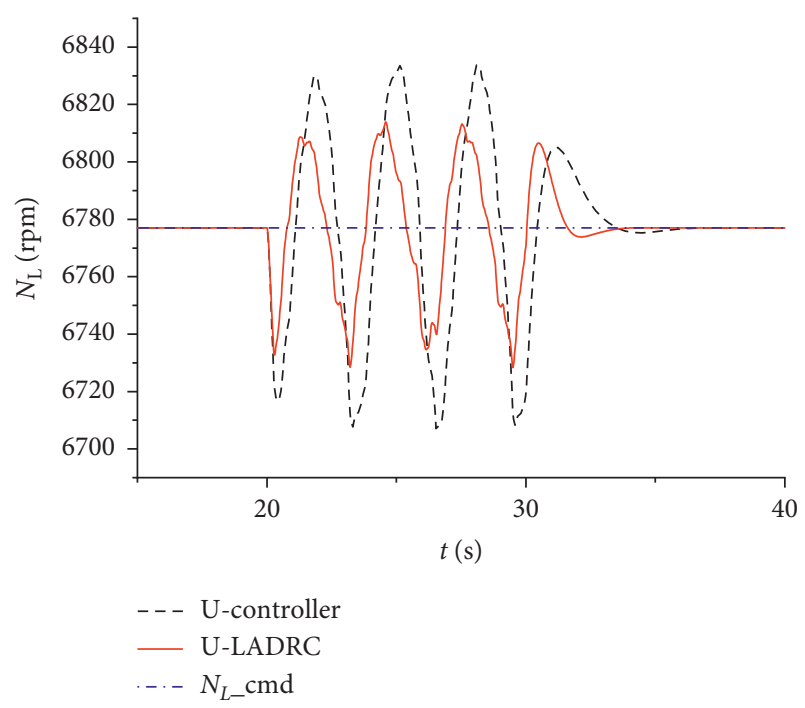

FIgURE 9: Antidisturbance effect of U-LADRC controller and Ucontroller in atmospheric disturbance.

compensation from the calculation of the LADRC compensator. The steady-state maximum relative variation decreases from $1.10 \%$ to $0.74 \%$.

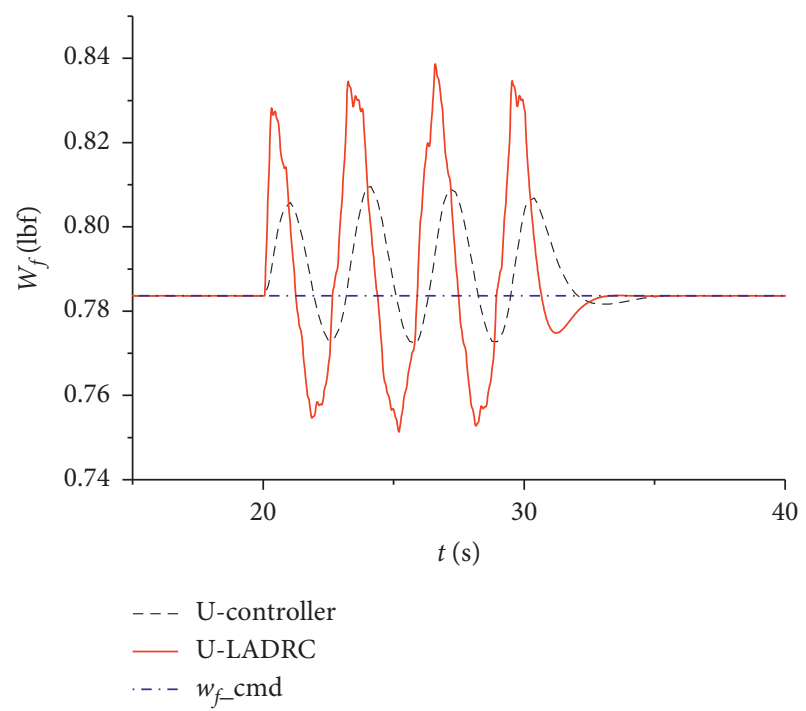

Figure 10: Fuel flow changing curve of U-LADRC controller and U-controller.

4.2.2. Power Extraction Disturbance. When the aeroengine is operating at the steady state in cruise, the electrical components on the aircraft (such as motors/generators and battery packs) may extract a certain amount of engine shaft power for power generation and storage of electrical energy $[21,22]$. Power extraction can ensure the entire aircraft power energy of the system, but it is also a kind of disturbance in flight that will affect the smooth flight of the aircraft. Power extraction disturbance schematic diagram is simply shown in Figure 11.

When the HP shaft power extraction disturbance occurs, the rotor dynamic equation of HP shaft becomes

$$
\frac{\mathrm{d} N_{H}}{\mathrm{~d} t}=\left(\frac{30}{\pi}\right)^{2} \frac{1}{N_{H}} \frac{1}{J_{H}}\left(\eta_{h} P_{\mathrm{HPT}}-P_{\mathrm{HPC}}-\Delta P_{m}\right) .
$$

The disturbance in $N_{H}$ will affect $N_{L}$ too. This section studies the antidisturbance effect of U-LADRC controller on the LP rotor speed under the HP shaft power extraction disturbance. In the digital simulation, a certain amount of horsepower is extracted from the HP shaft in $20 \sim 40 \mathrm{~s}$. The power extraction plan is shown in Figure 12. During the power extraction period, the antidisturbance effect of 


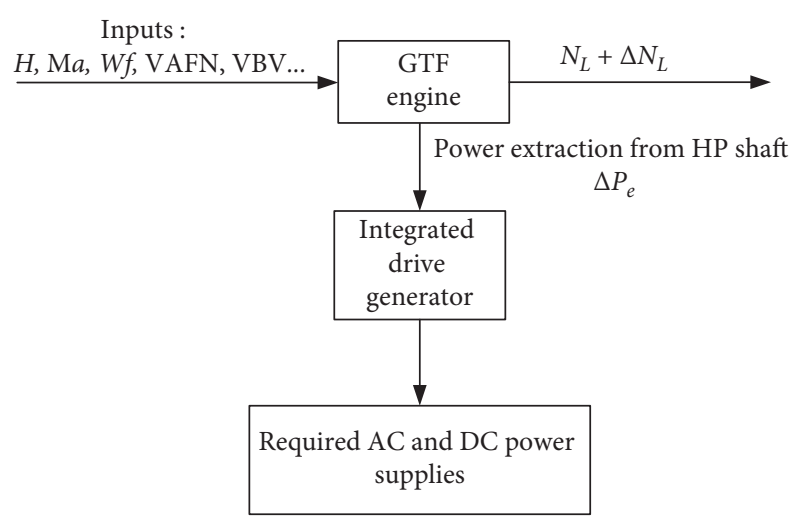

FIgUre 11: Power extraction disturbance schematic diagram.

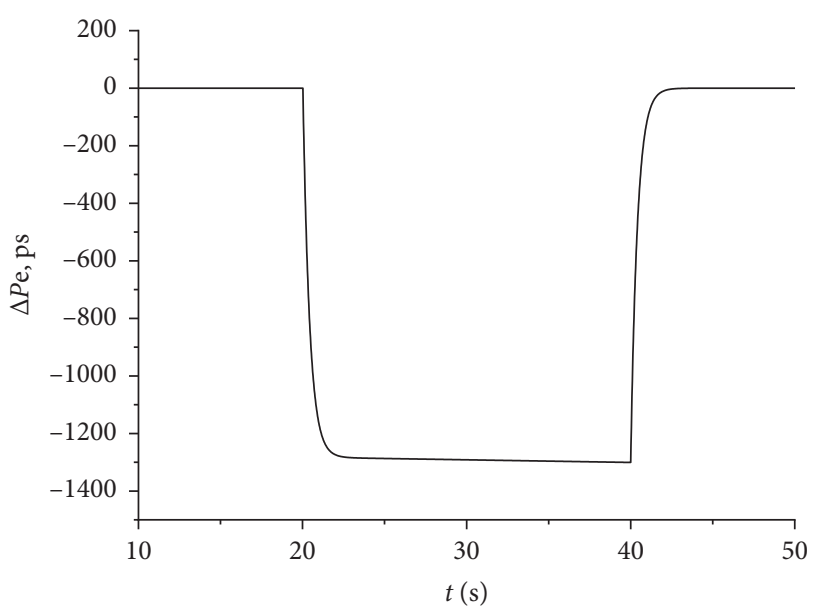

Figure 12: Power extraction plan in the HP shaft.

U-LADRC controller and U-controller is shown in Figure 13. The fuel flow changing curve of U-LADRC controller and U-controller is shown in Figure 14.

From Figures 13 and 14, we can see that the LADRC compensator plays the role of advanced compensation. It greatly improves the antidisturbance performance. The steady-state maximum relative variation decreases from $1.62 \%$ to $0.59 \%$.

\section{Verification in HIL Platform}

At present, in the development of aeroengine control system, the cycle iterative design approach of "all-digital simulation," "hardware-in-the-loop simulation (HIL)," "semiphysical simulation," "engine platform test," and "flight verification" is adopted, which can shorten the development cycle and reduce the design cost. Since the all-digital simulation is only a preliminary test of the control algorithm, the real-time performance of the controller cannot be verified. Therefore, with the characteristics of low difficulty, relatively low cost, and closeness to the actual operating environment of the engine, the hardware-in-the-loop simulation becomes the most important part in the development of the whole engine control system. It is very necessary to carry out the verification of controller in HIL platform.

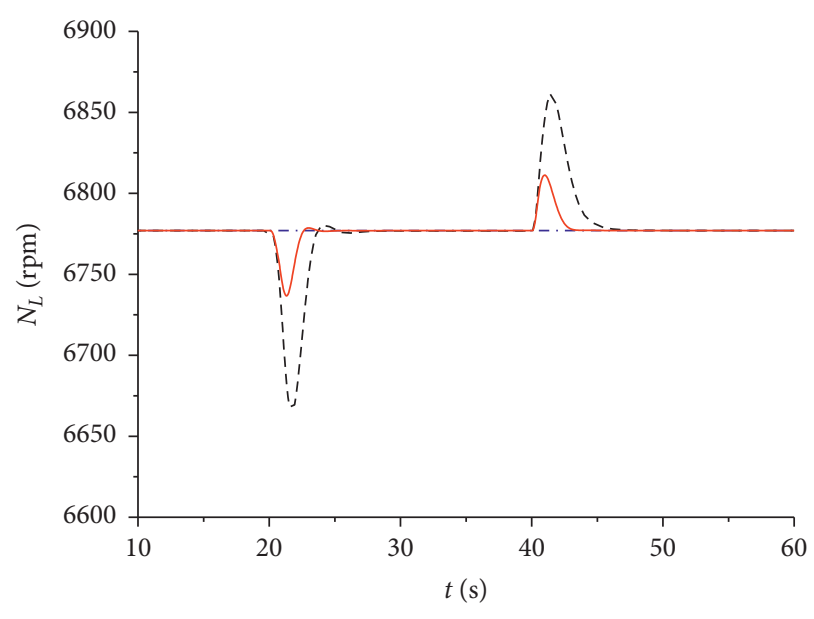

$$
\begin{array}{ll}
- & N_{L-c m d} \\
--- & \text { U-controller } \\
& \text { U-LADRC }
\end{array}
$$

FIGURE 13: Antidisturbance effect of U-LADRC controller and Ucontroller.

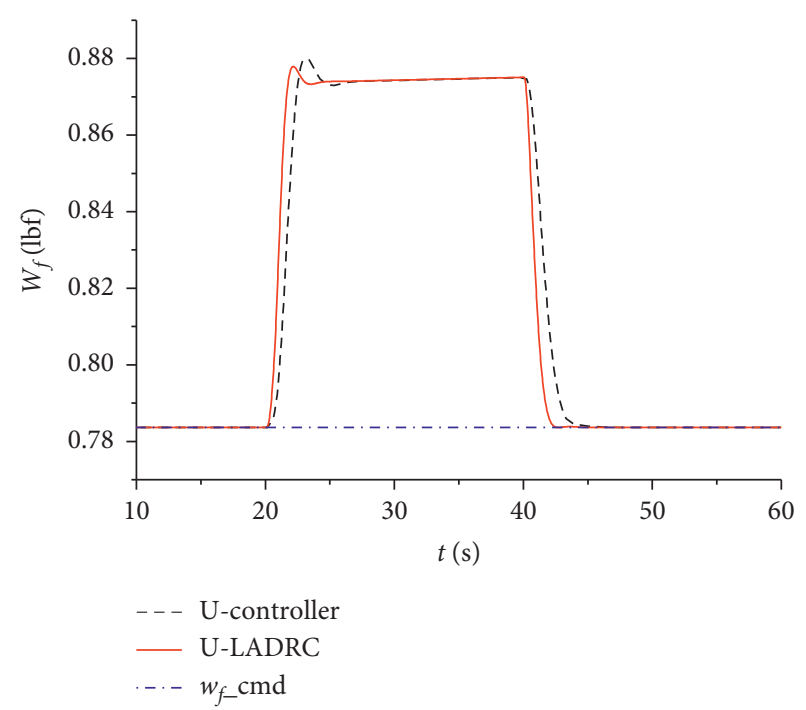

FIGURE 14: Fuel flow changing curve of U-LADRC controller and U-controller.

5.1. Introduction of HIL Platform. Aeroengine/gas turbine control system hardware-in-the-loop platform (hereinafter referred to as HIL platform) in Nanjing University of Aeronautics and Astronautics provides a complete integration and verification process for the engine model and control algorithm. The physical platform is shown in Figure 15.

The HIL platform has 3 parts: monitoring console, simulator, and controller. Their functions are introduced as follows:

Monitoring console: monitoring, operation management, and fault injection of the entire hardware-in-theloop platform. 

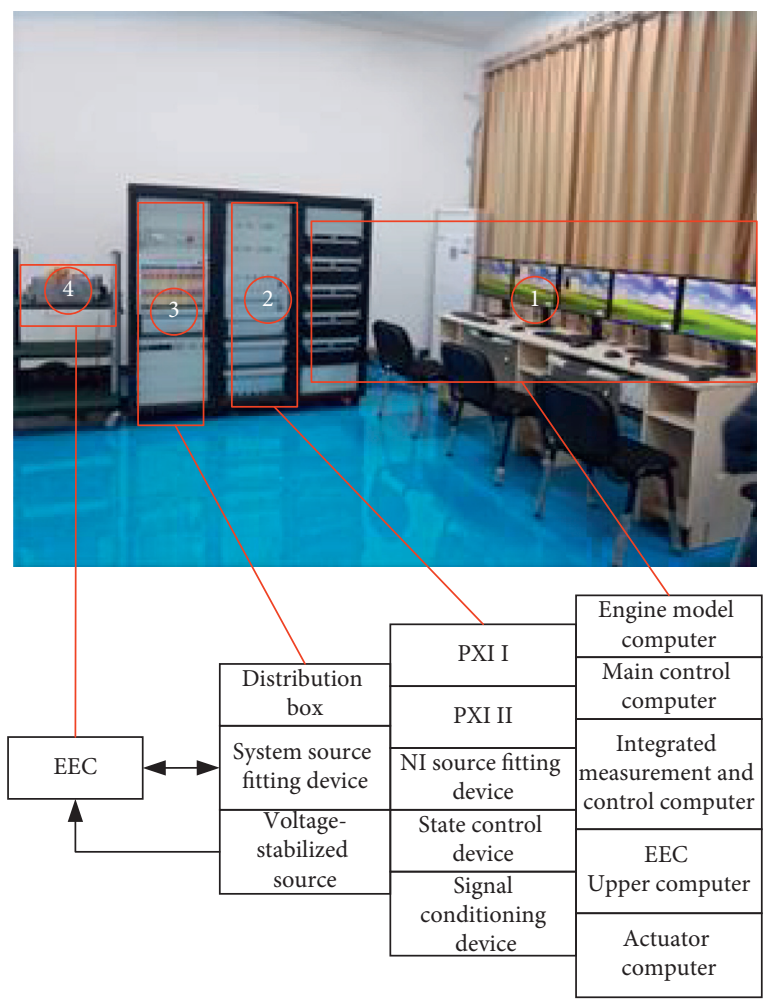

FIGURE 15: Aeroengine/gas turbine control system HIL platform in Nanjing University of Aeronautics and Astronautics.

Simulator: simulating the performance of the engine/ actuator in the entire envelope, transmitting the analog signal to the digital controller, and receiving the control signal output by the controller.

Controller: consisting of EEC and EEC upper computer. EEC operates the control algorithm code to control the engine model and EEC upper computer monitors various parameters of EEC in real time.

The data flow diagram of the HIL platform is shown in Figure 16. The entire simulation process is a closed-loop structure. The engine model calculates the output speed, temperature, pressure, and other signals. The PXI industrial computer measures these signals and sends them to the signal conditioning device. After receiving the signals, signal conditioning device transmits them to the controller through the system source fitting device. The controller calculates the value of control signals through algorithms and sends these signals such as fuel flow to the engine model through the actuator model.

5.2. Code Generation and Verification Process. In this paper, model-based design (MBD) method [23] is used to verify the control and antidisturbance performance of the U-LADRC controller. The entire code generation and integration process in the HIL platform is shown in Figure 17. First, in the Simulink environment, the designed controller and GTF engine model in Section 4 are packaged and checked according to the Simulink automatic code generation specification, and the corresponding code is generated. The

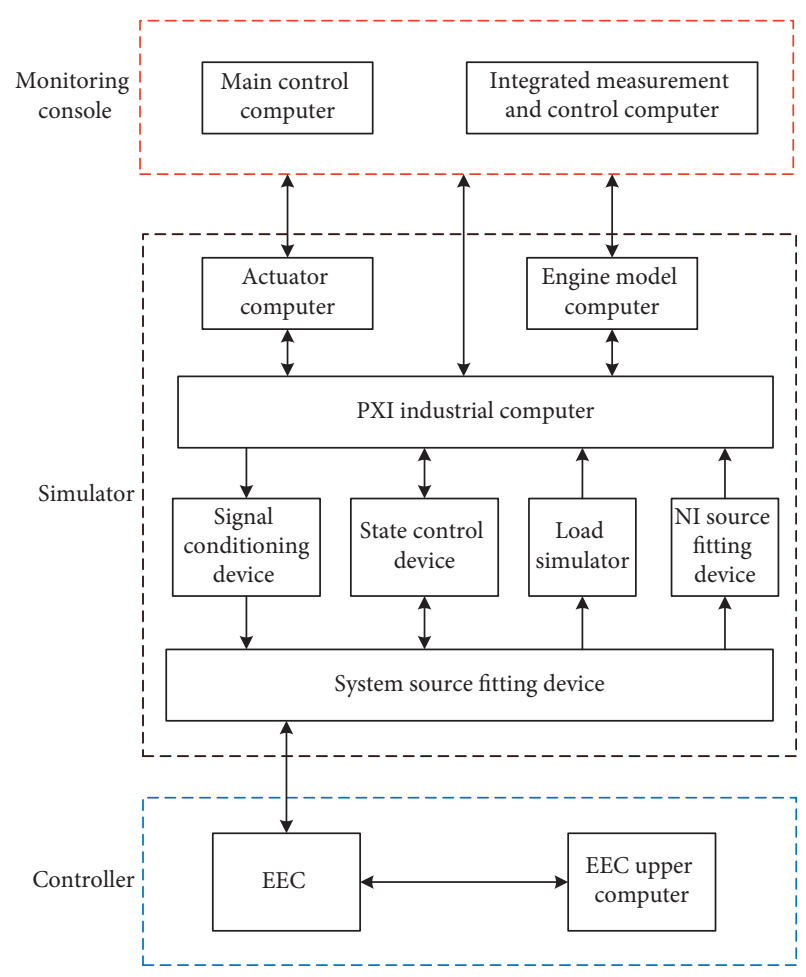

FIgURE 16: Date flow diagram of the HIL platform.

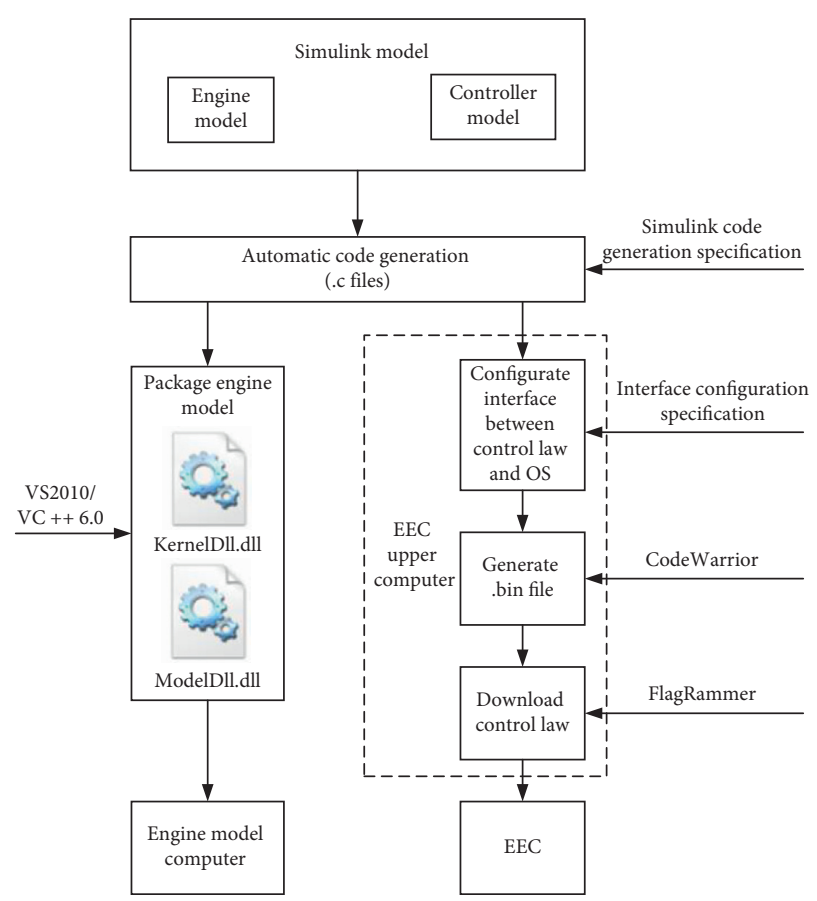

FIgURE 17: Process of code generation and integration in the HIL platform.

code of the engine model is packaged into a.dll file after twolayer integration; then, they are put into the engine model computer. The code of the controller is imported into the EEC host computer for interface configuration. When interface configuration is finished, the bin file can be generated and imported into the EEC. Then, the closed-loop 


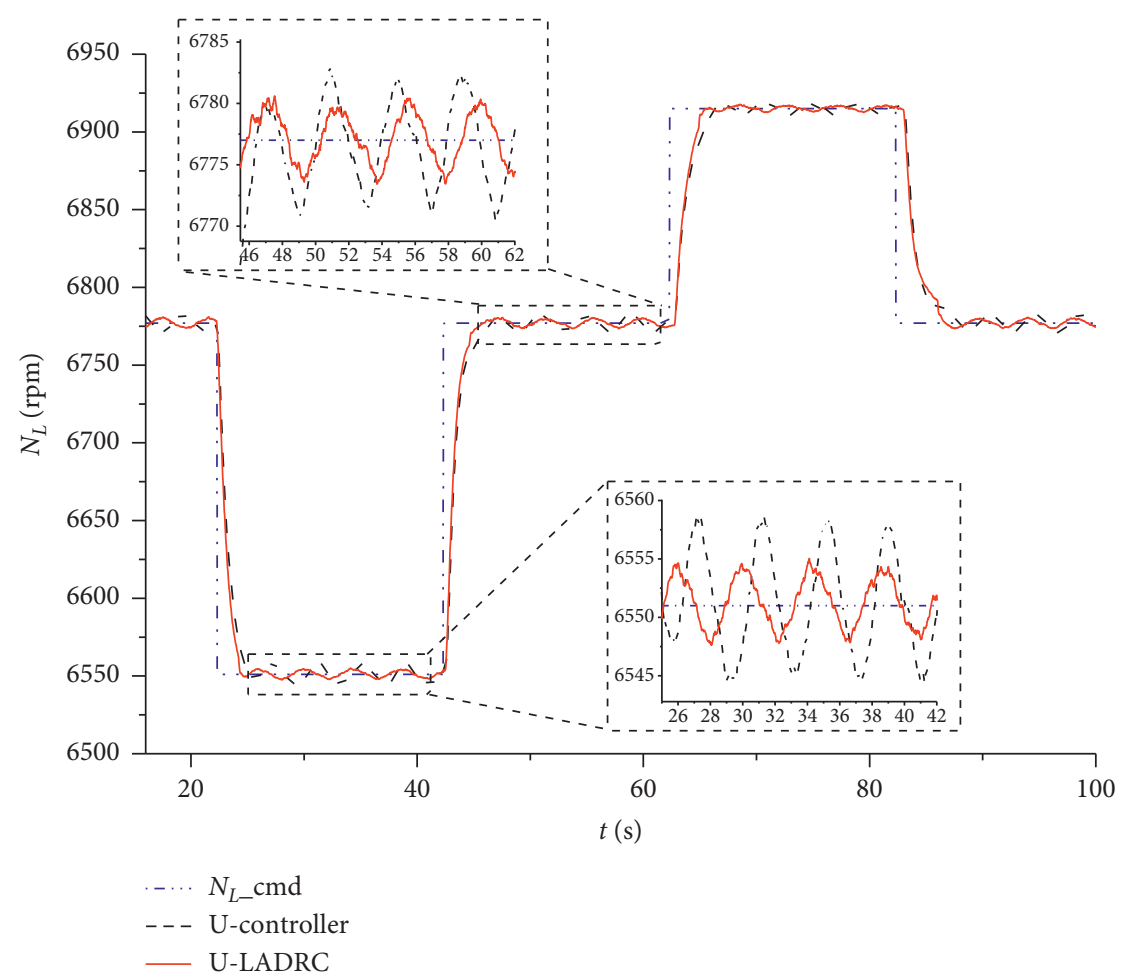

FIGURE 18: The transient-state response performance in the HIL platform.

verification test of the control algorithms under the HIL platform can be performed.

5.3. Result Analysis. In the HIL verification test, the same test example in the digital simulation platform is used to verify the transient-state response performance and the antidisturbance performance of the U-LADRC controller.

The transient-state response performance in the HIL platform is shown in Figure 18. Because there are many uncertain factors such as noise and voltage fluctuations in the hardware transmission link of the entire HIL platform, the sensor signal and the actuator signal may be inaccurate. These unavoidable hardware disturbances will affect the transient and steady performance, which are not considered in the digital simulation platform. In the HIL verification, the U-model controller without the LADRC compensator is used as a comparison. The experimental results show that the U-LADRC controller has a speed jitter amplitude of $\pm 4 \mathrm{rpm}$ in the steady state of the HIL environment, which is lower than $\pm 7 \mathrm{rpm}$ in $\mathrm{U}$-model control without the LADRC compensator. U-LADRC controller has a better antidisturbance performance.

U-LADRC controller's antidisturbance performance against atmospheric disturbance and power extraction disturbance is verified in the HIL platform, too. The results are shown in Figures 19 and 20.

During the whole experimental process, the uncertain disturbance effect of the HIL platform itself is superimposed simultaneously. In order to quantitatively characterize the improvement of the antidisturbance performance before

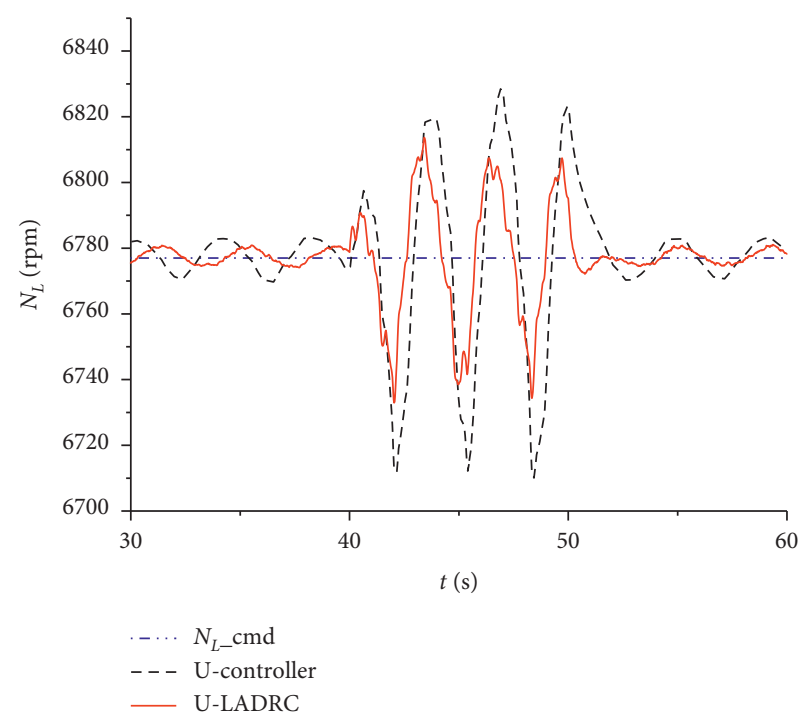

Figure 19: Antidisturbance effect under atmospheric disturbance in the HIL platform.

and after the addition of the LADRC compensator, the integration of absolute error (IAE) criterion is used. Performance index $J$ is defined as

$$
J=\int_{t_{1}}^{t_{2}}\left|N_{L_{-} \text {real }}-N_{L_{-} s}\right| \mathrm{d} t,
$$

where $N_{L_{-} \text {real }}$ represents the real LP rotor speed and $N_{L_{-} s t}$ represents the command of LP rotor speed. The selection of $\left[t_{1}, t_{2}\right]$ needs to include the time before and after the disturbance occurs. Assume that $t_{b}$ is the beginning time of 


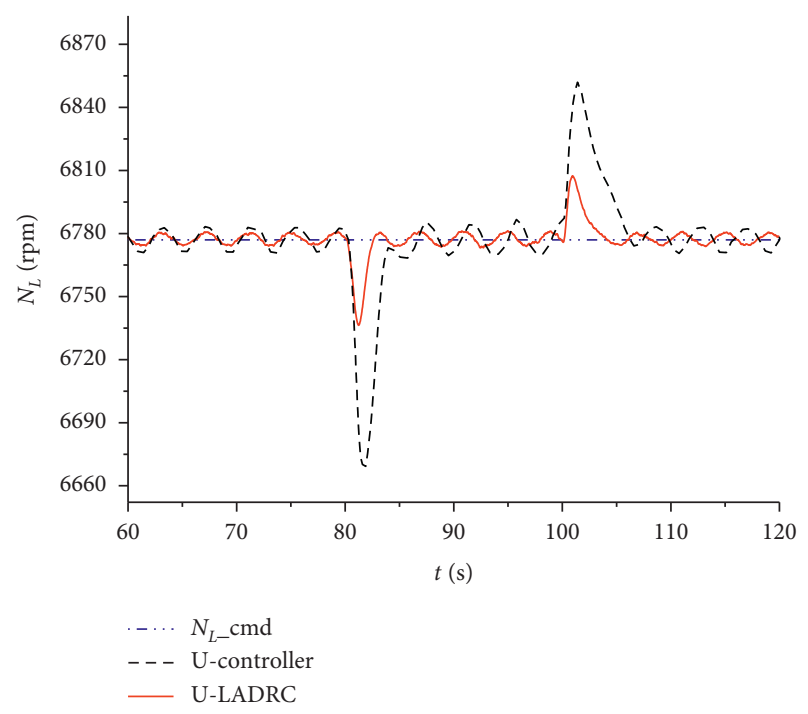

FIgURe 20: Antidisturbance effect under power extraction atmospheric disturbance in the HIL platform.

TABLE 1: Comparison of antidisturbance performance.

\begin{tabular}{lcc}
\hline & $J_{a}$ & $J_{p}$ \\
\hline U-controller & 415.3867 & 649.7069 \\
U-LADRC & 229.1846 & 204.4373 \\
Improvement (\%) & 44.8 & 68.5 \\
\hline
\end{tabular}

the disturbance and $t_{e}$ is the end time of the disturbance, and two kinds of $J$ are defined:

$$
\begin{aligned}
& J_{a}=\int_{t_{b}-10}^{t_{e}+10}\left|N_{L \_ \text {real }}-N_{L_{-s} t}\right| \mathrm{d} t, \\
& J_{p}=\int_{t_{b}-20}^{t_{e}+20}\left|N_{L_{-} \text {real }}-N_{L_{-} s}\right| \mathrm{d} t,
\end{aligned}
$$

where $J_{a}$ represents the antidisturbance performance under atmospheric disturbance and $J_{p}$ represents the antidisturbance performance under power extraction disturbance. Smaller $J$ means better antidisturbance performance. The value of two kinds of $J$ in the HIL platform test before and after the addition of the LADRC compensator is shown in Table 1.

It can be seen that the LADRC compensator greatly improves the antidisturbance performance of the U-controller.

\section{Results}

In this paper, a novel rotor speed controller was designed for a GTF engine and both digital simulation and HIL platform verification have been performed. Results are summarized as follows:

(1) A U-control method based on the aeroengine LPV model is proposed for the first time and applied to aeroengine low-pressure speed transient and steady control. While guaranteeing the good control performance, it greatly simplifies the complex design process of the traditional LPV gain scheduling controller.

(2) Aiming at the problem of poor antidisturbance performance of the U-controller designed in (1), the U-LADRC controller is designed. In this controller, a LADRC compensator is added to make up for the bad effects of disturbances in advance without changing the original U-model control structure.

(3) The LADRC compensator has a significant antidisturbance performance on various types of disturbances. Experiments results show that the antidisturbance performance of U-LADRC rotor speed controller is more than $40 \%$ higher than that of the U-controller without the LADRC compensator.

(4) The U-LADRC rotor speed controller is successfully integrated and verified on the HIL platform to meet the real-time requirement of real EEC conditions. It has a great practical application potential.

\section{Data Availability}

The data used to support the findings of this study are available from the corresponding author upon request.

\section{Conflicts of Interest}

The authors declare that they have no conflicts of interest regarding the publication of this paper.

\section{Acknowledgments}

The authors are grateful for the financial support by the Fundamental Research Funds for the Central Universities (no. NS2020017); the Central Military Commission Foundation to Strengthen Program Technology Fund (no. 2019JCJQ-JJ-347); Major Special Basic Research Projects for Aviation Engines \& Gas Turbines (no. 1002-DLJ19002); and Major Special Basic Research Projects for Green \& Sustainable Manufacturing (no. 1002-DCB16001).

\section{References}

[1] SAE International Group, "Electronic engine control specifications and standards," Report No.: AIR4250B, SAE International Group, Warrendale, PA, USA, 2012.

[2] S. Q. Fan, Aircraft Engine Control, Northwestern Polytechnical University Press, Xi'an, China, 2008.

[3] Q. M. Zhu and L. Z. Guo, "A pole placement controller for non-linear dynamic plants," Proceedings of the Institution of Mechanical Engineers, Part I: Journal of Systems and Control Engineering, vol. 216, no. 6, pp. 467-476, 2002.

[4] F. X. Xu, Q. M. Zhu, and D. Y. Zhao, "U-model based design methods for nonlinear control systems a survey of the development in the 1st decade," Control and Decision, vol. 28, no. 7, pp. 961-971, 2013.

[5] W. Du, Q. M. Zhu, and X. Wu, "Support vector machine based U-model generalized predictive controller for nonlinear dynamic plants," in Proceedings of the 33th Chinese Control Conference, IEEE press, Nanjing, China, July 2014. 
[6] M. Shafiq and M. Haseebuddin, "U-model-based internal model control for non-linear dynamic plants," Proceedings of the Institution of Mechanical Engineers, Part I: Journal of Systems and Control Engineering, vol. 219, no. 6, pp. 449-458, 2005.

[7] X. Wu, L. Liu, Q. Zhu, W. Du, B. Wang, and J. Zhang, "Umodel-based adaptive control for a class of stochastic nonlinear dynamic plants with unknown parameters," International Journal of Modelling, Identification and Control, vol. 13, no. 3, pp. 135-143, 2011.

[8] E. Hasan, R. B. Ibrahim, S. S. A. Ali, K. Bingi, and S. F. Gilani, "U-model based adaptive control of gas process plant," Procedia Computer Science, vol. 105, pp. 119-124, 2017.

[9] J. Q. Han, "Auto disturbances rejection control technique," Frontier Science, vol. 1, pp. 24-31, 2007.

[10] Z. Q. Gao, "Active disturbance rejection control: a paradigm shift in feedback control system design," in Proceedings of the 2006 American Control Conference, pp. 2399-2405, Minneapolis, MN, USA, June 2006.

[11] J. Huang, S. Ri, T. Fukuda, and Y. Wang, "A disturbance observer based sliding mode control for a class of underactuated robotic system with mismatched uncertainties," IEEE Transactions on Automatic Control, vol. 64, no. 6, pp. 2480-2487, 2019.

[12] J. Wang, M. Zhang, S. Ri, C. Xiong, Z. Li, and Y. Kang, "Highorder disturbance-observer-based sliding mode control for mobile wheeled inverted pendulum systems," IEEE Transactions on Industrial Electronics, vol. 67, no. 3, pp. 2030-2041, 2020.

[13] H. B. Zhang and J. G. Sun, "Application of active disturbance rejection control method in aeroengine afterburning transition state control," Journal of Propulsion Technology, vol. 31, no. 2, pp. 219-225, 2010.

[14] Q. M. Zhu, W. C. Zhang, and J. Na, "U-model based control design framework for continuous-time systems," in Proceedings of the Chinese Control Conference, Wuhan, China, July 2019.

[15] D. Yuan, X. J. Ma, and Q. H. Zeng, "Research on frequencyband characteristics and parameters configuration of linear active disturbance rejection control for second-order systems," Control Theory \& Applications, vol. 30, pp. 1630-1640, 2013.

[16] Z. Q. Chen, M. W. Sun, and R. G. Yang, "On the stability of linear active disturbance rejection control," Acta Automatica Sinica, vol. 39, no. 5, pp. 574-580, 2014.

[17] X. Chen, D. H. Li, and Z. Q. Gao, "Tuning method for secondorder active disturbance rejection control," in Proceedings of the 30th Chinese Control Conference, pp. 6322-6327, Yantai, China, July 2011.

[18] J. W. Chapman and S. L. Litt, "Control design for an advanced geared turbofan engine,” Report No.: NASA/TM-219569, 2017.

[19] C. Kravaris and R. A. Wright, "Deadtime compensation for nonlinear processes," AICHE Journal, vol. 35, no. 9, pp. 1537-1542, 1989.

[20] G. Kopasakis, "Atmospheric turbulence modeling for aero vehicles: fractional order fits," Report No.: NASA/TM216961, 2010.

[21] J. Mcnichols, C. Barnes, and M. Wolff, "Hardware-in-the-loop power extraction using different real-time platforms," Nature Biotechnology, vol. 21, no. 4, pp. 422-427, 2013.

[22] S. J. Khalid, "Mitigating impact of bleed and power extraction with more electric architectures," in Proceedings of the SAE 2016 Aerospace Systems and Technology Conference, Hartford, CT, USA, September 2016.
[23] F. R. Liu, "Model-based developing approach for airborne software of commercial engine," Process Automation Instrumentation, vol. 38, no. 6, pp. 26-30, 2017. 